\title{
Bounds for Effective Parameters of Heterogeneous Media by Analytic Continuation
}

\author{
K. Golden` and G. Papanicolaou ${ }^{\star}$ \\ Courant Institute, New York University, New York, NY 10012, USA
}

\begin{abstract}
We give a mathematical formulation of a method for obtaining bounds on effective parameters developed by D. Bergman and G. W. Milton. This method, in contrast to others used before, does not rely on a variational principle, but exploits the properties of the effective parameter as an analytic function of the component parameters. The method is at present restricted to two-component media.
\end{abstract}

\section{Introduction}

Once the notion of an effective parameter of a composite or heterogeneous medium has been properly established, it becomes clear that even in simple cases, for example in periodic structures, these effective parameters are very difficult to calculate. Consequently one turns to the determination of qualitative properties such as bounds for these parameters under various assumptions about the microscopic properties of the medium under study.

Recently Bergman [1-6] introduced a method for obtaining bounds which does not rely on the availability of variational principles. The method of Bergman has been elaborated upon in detail and applied to several problems by G. W. Milton [710]. Bounds obtained previously, in particular the Hashin-Shtrikman bounds [1113] required variational principles. The scope and utility of variational methods for obtaining bounds is analyzed in [14], while extensive analysis and use of variational methods has been made by Willis $[15,16]$, Dedericks and Zeller [17], as well as many other authors referred to in these papers.

Our purpose here is to give a mathematical basis for Bergman's method, in particular for the key step in it which is a representation formula for the effective parameter. This formula is restricted at present to two-component inhomogeneous media.

* Supported by a Hertz Foundation fellowship

$\star \star$ Research supported by the Air Force Office of Scientific Research under Grant AFOSR-80-0228 
The reason the representation formula is important is as follows. When the microscopic medium is nearly homogeneous (its parameters differ little from constant values), one can derive approximations for the effective parameters by direct perturbation analysis. The information contained in the perturbation expansion can then be used together with the representation formula to yield global results not restricted to nearly homogeneous media.

Our interest in Bergman's method was stimulated by the very interesting lectures of Bergman and Milton given during a meeting in New York in June 1981 and by discussions with Luc Tartar who has yet another very interesting method for obtaining bounds without the use of variational principles.

\section{Formulation of the Problem}

We shall consider the determination of bounds for the effective dielectric constant (or conductivity) of a two or several-component microscopically isotropic material. For the formulation of effective parameters we shall consider a general dielectric (or conducting) medium.

Let $(\Omega, \mathscr{F}, P)$ be a probability space and let $\varepsilon_{i j}(x, \omega)$ be strictly stationary random fields of $x \in \mathbb{R}^{d}, i, j=1,2, \ldots, d$ such that

$$
\alpha \sum_{i=1}^{d} \xi_{i}^{2} \leqq \sum_{i, j=1}^{d} \varepsilon_{i j}(x, \omega) \xi_{i} \xi_{j} \leqq \beta \sum_{i=1}^{d} \xi_{i}^{2},
$$

where $\alpha$ and $\beta$ are constants independent of $x$ and $\omega$ and $\xi_{i} \in \mathbb{R}, i=1, \ldots, d$. By strict stationarity we mean that the joint distribution of $\varepsilon_{i j}\left(x_{1}, \omega\right)$, $\varepsilon_{i j}\left(x_{2}, \omega\right), \ldots, \varepsilon_{i j}\left(x_{N}, \omega\right)$ for any points $x_{1}, x_{2}, \ldots, x_{N} \in \mathbb{R}^{d}$ and that of $\varepsilon_{i j}\left(x_{1}+h, \omega\right), \ldots, \varepsilon_{i j}\left(x_{N}+h, \omega\right)$ for any $h \in \mathbb{R}^{d}$ are the same. More specifically, we shall assume as in [18] that there is a group of transformations $\tau_{x}, x \in \mathbb{R}^{d}$, from $\Omega$ into $\Omega$ that is one to one and preserves the measure $P$. That is, $\tau_{x} \tau_{y}=\tau_{x+y}$ and for any $A \in \mathscr{F}$, $P\left(\tau_{-x} A\right)=P(A)$. We shall suppose that there are measurable functions $\tilde{\varepsilon}_{i j}(\omega)$ on $\Omega$ satisfying (2.1) such that

$$
\varepsilon_{i j}(x, \omega)=\tilde{\varepsilon}_{i j}\left(\tau_{-x} \omega\right), x \in \mathbb{R}^{d}, \omega \in \Omega, i, j=1,2, \ldots, d .
$$

We consider the following problem in electrostatics. Find two stationary random vector fields $E_{i}(x, \omega)$ and $D_{i}(x, \omega), i=1,2, \ldots, d$ such that

$$
\begin{gathered}
D_{i}(x, \omega)=\sum_{j=1}^{d} \varepsilon_{i j}(x, \omega) E_{j}(x, \omega), \quad i=1,2, \ldots, d, \\
\frac{\partial}{\partial x_{j}} E_{i}(x, \omega)=\frac{\partial}{\partial x_{i}} E_{j}(x, \omega) \quad i, j=1,2, \ldots, d, \\
\sum_{j=1}^{d} \frac{\partial}{\partial x_{j}} D_{j}(x, \omega)=0, \\
\int_{\Omega} P(d \omega) E_{i}(x, \omega)=\bar{E}_{i}, \quad i=1,2, \ldots, d .
\end{gathered}
$$

The constant vector $\bar{E}$ is given. The existence of a unique solution to (2.3)-(2.6) is proved in the next section since the setup of the proof will be needed for the 
representation formula we want. Once $E_{i}$ and $D_{i}$ have been obtained, the effective dielectric constant $\varepsilon_{i j}^{*}$ is defined by

$$
\sum_{j=1}^{d} \varepsilon_{i j}^{*} \bar{E}_{j}=\int_{\Omega} P(d \omega) D_{i}(x, \omega), \quad i=1,2, \ldots, d .
$$

Problem (2.3)-(2.6) is the usual electrostatic problem for the electric field $E$ and the dielectric displacement $D$ (or the negative temperature gradient and the heat flux respectively for the thermal conductivity problem) in a stationary random dielectric medium when the mean electric field $\bar{E}$ is a given constant vector. The average dielectric displacement for unit mean electric field is the effective dielectric constant as expressed by (2.7) and is independent of $x \in \mathbb{R}^{d}$.

In the appendix, we show that the above definition coincides with the usual and perhaps more intuitive one. There, a finite cube of dielectric material with edges of length $2 N$ is considered and the average dielectric displacement $D_{i}^{(N)}$ is computed under suitable boundary conditions. If $\varepsilon_{i j}^{*, N}(\omega)$ denotes the average dielectric displacement in the $i^{\text {th }}$ direction due to a unit mean electric field in the $j^{\text {th }}$ direction, we show that as $N \rightarrow \infty$ it tends to the $\varepsilon_{i j}^{*}$ of (2.7), which is what one expects.

The reason that we consider an infinite stationary medium and work with it directly is because $\varepsilon_{i j}^{*}$ is a local quantity that has nothing to do with macroscopic boundaries. Moreover, the finiteness of a domain conflicts with the properties of stationarity and isotropy which simplify the analysis considerably.

The microscopic dielectric constant $\varepsilon$ (or tensor $\varepsilon_{i j}$ ) must be positive in accordance with (2.1). In some situations the fields as well as $\varepsilon$ can be complexvalued and (2.3)-(2.6) is still well posed. Complex dielectric constants arise from the time harmonic form of Maxwell's equations when $\varepsilon$ is replaced by $\varepsilon+\sigma / i \gamma$, where $\sigma$ is the conductivity and $\gamma$ is the radian frequency of oscillation of the fields.

The object of this paper is the determination of the range of values of $\varepsilon_{i j}^{*}$ under various hypotheses on $\varepsilon_{i j}(x, \omega)$. We return to this in Sects. 5 and 6 .

\section{Existence and uniqueness for the Electrostatic Problem}

We shall show that (2.3)-(2.6) suitably formulated has a unique stationary solution $E_{i}(x, \omega), D_{i}(x, \omega)$. The analysis is similar to the one in [18].

The group of transformations $\tau_{x}$ acting on $\Omega$ induces a group of operators on the Hilbert space of real-valued functions $H=L^{2}(\Omega, \mathscr{F}, P)$ with inner product

$$
(\widetilde{f}, \tilde{g})=\int_{\Omega} P(d \omega) \tilde{f}(\omega) \tilde{g}(\omega), \quad \tilde{f}, \tilde{g} \in H .
$$

The group of operators $T_{x}$ on $H$ is given by

$$
\left(T_{x} \tilde{f}\right)(\omega)=\tilde{f}\left(\tau_{-x} \omega\right), \quad x \in \mathbb{R}^{d}
$$

Since $\tau_{x}$ is measure preserving, the operators $T_{x}$ form a unitary group. Therefore they have closed densely defined infinitesimal generators $L_{i}$ in each direction $i=1,2, \ldots, d$, with domain $\mathscr{D}_{i} \subset H$. Thus

$$
L_{i}=\left.\frac{\partial}{\partial x_{i}} T_{x}\right|_{x=0}, \quad i=1,2, \ldots, d,
$$


with differentiation defined in the sense of convergence in $H$ for elements of $\mathscr{D}_{i}$. The closed subset of $H$

$$
\mathscr{D}=\bigcap_{i=1}^{d} \mathscr{D}_{i}
$$

becomes a Hilbert space with the inner product

$$
(\tilde{u}, \tilde{v})=\int_{\Omega} P(d \omega) \tilde{u}(\omega) \tilde{v}(\omega)+\sum_{i=1}^{d} \int_{\Omega} P(d \omega) L_{i} \tilde{u}(\omega) L_{i} \tilde{v}(\omega) .
$$

We return to (2.3)-(2.6). Since everything involved with this problem is to be stationary, we look for solutions $E_{i}, D_{i}$ in the form

$$
E_{i}(x, \omega)=\tilde{E}_{i}\left(\tau_{-x} \omega\right), \quad D_{i}(x, \omega)=\tilde{D}_{i}\left(\tau_{-x} \omega\right) .
$$

In fact, let $\mathscr{H}$ be the Hilbert space

$$
\mathscr{H}=\left\{\widetilde{f}_{i}(\omega) \in H, i=1,2, \ldots, d \mid L_{i} \widetilde{f}_{j}=L_{j} \widetilde{f}_{i} \text { weakly and } \int_{\Omega} \tilde{f}_{i}(\omega) P(d \omega)=0\right\},
$$

and consider the following variational problem: find $\tilde{G}_{i}(\omega)$ in $\mathscr{H}$ such that

$$
\int_{\Omega} P(d \omega) \sum_{i, j=1}^{d} \tilde{\varepsilon}_{i j}(\omega)\left(\tilde{G}_{j}(\omega)+\bar{E}_{j}\right) \tilde{f}_{i}(\omega)=0
$$

for all $f_{i} \in \mathscr{H}$. In view of $(2.1)$ this problem has a unique solution by the Lax-Milgram lemma. Clearly

$$
\tilde{E}_{i}(\omega)=\widetilde{G}_{i}(\omega)+\bar{E}_{i}, \tilde{D}_{i}(\omega)=\sum_{j=1}^{d} \tilde{\varepsilon}_{i j}(\omega) \tilde{E}_{j}(\omega)
$$

is the unique solution of (2.3)-(2.6) via (3.5).

The effective dielectric constant defined by (2.7) can also be written in the form

$$
\sum_{j=1}^{d} \varepsilon_{i j}^{*} \bar{E}_{j}=\sum_{j=1}^{d} \int_{\Omega} P(d \omega) \tilde{\varepsilon}_{i j}(\omega) \tilde{E}_{j}(\omega)
$$

Choosing the components of the constant vector $\bar{E}_{j}$ equal to $\delta_{j k}, j=1,2, \ldots, d$ for some $k=1,2, \ldots, d$, and denoting the corresponding field $\widetilde{E}$ by $\widetilde{E}_{j}^{k}$, (3.9) is transformed to

$$
\varepsilon_{i k}^{*}=\sum_{j=1}^{d} \int_{\Omega} P(d \omega) \tilde{\varepsilon}_{i j}(\omega) \tilde{E}_{j}^{k}(\omega) \quad i, k=1,2, \ldots, d .
$$

In view of (3.7) we can write (3.10) also in the symmetric form

$$
\varepsilon_{l k}^{*}=\sum_{i, j=1}^{d} \int_{\Omega} P(d \omega) \tilde{\varepsilon}_{i j}(\omega) \tilde{E}_{j}^{k}(\omega) \tilde{E}_{i}^{l}(\omega), \quad l, k=1,2, \ldots, d .
$$

One can equally well formulate (2.3)-(2.5) with (2.6) replaced by

$$
\int_{\Omega} P(d \omega) D_{i}(x, \omega)=\bar{D}_{i}, \quad i=1,2, \ldots, d,
$$


where now we focus attention on the $D$ field and find a suitable Hilbert space for it (analog of $\mathscr{H}$ in (3.6) but "divergence free" vectors now) and a suitable variational equation analogous to (3.7). This is quite straightforward, the principal conclusion being that the tensor $\left(\varepsilon^{*}\right)_{i j}^{-1}$ is given by

$$
\sum_{j=1}^{d}\left(\varepsilon^{*}\right)_{i j}^{-1} \bar{D}_{j}=\sum_{j=1}^{d} \int P(d \omega)(\tilde{\varepsilon})_{i j}^{-1}(\omega) \widetilde{D}_{j}(\omega) .
$$

\section{The Representation Formula for Two Component Media}

Problem (2.3)-(2.6) was put in the variational form (3.7) over the Hilbert space $\mathscr{H}$ defined by (3.6). Let us restrict attention to a two-component medium of the form

$$
\varepsilon_{i j}(x, \omega)=\varepsilon(x, \omega) \delta_{i j},
$$

where $\varepsilon(x, \omega)$ takes only two values $\varepsilon_{1}$ and $\varepsilon_{2}$ with $0<\varepsilon_{1}<\varepsilon_{2}<\infty$. We write

$$
\varepsilon(x, \omega)=\varepsilon_{1} \chi_{1}(x, \omega)+\varepsilon_{2} \chi_{2}(x, \omega),
$$

where $\chi_{j}(x, \omega), j=1,2$, is the indicator function of the region occupied by the dielectric material labeled by $j$. In the notation of Sect. 3 we put

$$
\tilde{\varepsilon}(\omega)=\varepsilon_{1} \tilde{\chi}_{1}(\omega)+\varepsilon_{2} \tilde{\chi}_{2}(\omega),
$$

where, for example, $\tilde{\chi}_{1}(\omega)=1$ for all realizations $\omega \in \Omega$ for which the origin is occupied by dielectric material of type one and equals zero otherwise.

From (4.3) and the definition of $\widetilde{E}_{i}$ in (3.7) and (3.8), it follows that the field depends only on the ratio

$$
h=\frac{\varepsilon_{2}}{\varepsilon_{1}}
$$

Hence, from (3.10) we have

$$
\varepsilon_{i k}^{*}=\varepsilon_{1} \int_{\Omega} P(d \omega)\left(\tilde{\chi}_{1}(\omega)+h \tilde{\chi}_{2}(\omega)\right) \tilde{E}_{i}^{k}(\omega), \quad i, k=1,2, \ldots, d,
$$

so that $\varepsilon_{i k}^{*}=\varepsilon_{i k}^{*}(h)$. Our first observation about it is that

$\varepsilon_{i k}^{*}(h)$ is an analytic function of the complex variable

$h$ everywhere except on the negative real axis.

To prove this statement we look at the quadratic form associated with (3.7) and seek an $\alpha>0$ such that

$$
\left.\left.\left|\int_{\Omega} P(d \omega)\left(\tilde{\chi}_{1}+h \tilde{\chi}_{2}\right) \sum_{j=1}^{d}\right| \tilde{G}_{j}\right|^{2}\left|\geqq \alpha \int_{\Omega} P(d \omega) \sum_{j=1}^{d}\right| \tilde{G}_{j}\right|^{2},
$$

where $\widetilde{G}_{j}(\omega)$ is complex-valued when $h$ is complex. If we set

$$
\lambda=\frac{\int P(d \omega) \tilde{\chi}_{2} \sum_{j=1}^{d}\left|\tilde{G}_{j}\right|^{2}}{\int P(d \omega) \sum_{j=1}^{d}\left|\tilde{G}_{j}\right|^{2}}
$$


so that $0 \leqq \lambda \leqq 1$, the inequality (4.7) becomes

$$
|h \lambda+1-\lambda| \geqq \alpha .
$$

This means that (4.7) will hold for some $\alpha>0$ if and only if the origin is not on the line segment joining $h$ and 1, i.e. if and only if $h$ is not on the negative real axis including zero.

Thus for any complex $h$ off the negative real axis, (3.7) is coercive and by differentiation with respect to $h$ one deduces easily that $\widetilde{G}_{j}(\omega)$ is analytic in $h$ with values in $\mathscr{H}$. Consequently from $(3.10), \varepsilon_{i k}^{*}(h)$ is analytic in $h$ off the negative real axis.

Dividing (4.5) by $\varepsilon_{1}$ we write

$$
m_{i k}(h)=\varepsilon_{1}^{-1} \varepsilon_{i k}^{*}(h)=\int_{\Omega} P(d \omega)\left(\tilde{\chi}_{1}(\omega)+h \tilde{\chi}_{2}(\omega)\right) \tilde{E}_{i}^{k}(\omega),
$$

where $\tilde{E}_{i}^{k}(\omega)=\tilde{G}_{i}^{k}(\omega)+\delta_{i k}$ and (3.7) holds, or somewhat formally

$$
\sum_{i=1}^{d} L_{i}\left[\left(\tilde{\chi}_{1}(\omega)+h \tilde{\chi}_{2}(\omega)\right)\left(\tilde{G}_{i}^{k}(\omega)+\delta_{i k}\right)\right]=0, \quad k=1,2, \ldots, d .
$$

We are now ready to state the following

Representation Theorem. Let

$$
s=\frac{1}{1-h}, F_{i k}(s)=\delta_{i k}-m_{i k}(h) .
$$

There exist finite Borel measures $\mu_{i k}(d z)$ defined on $0 \leqq z \leqq 1$ such that the diagonals $\mu_{i i}(d z)$ are positive measures satisfying

$$
F_{i k}(s)=\int_{0}^{1} \frac{\mu_{i k}(d z)}{s-z}, \quad i, k=1,2, \ldots, d
$$

for all complex $s$ outside $0 \leqq \mathrm{Res} \leqq 1, \mathrm{Ims}=0$.

\section{Remarks}

1. In [1] Bergman assumes that $F_{11}(s)\left(\right.$ or any other $\left.F_{i i}(s) i=1,2, \ldots, d\right)$ is a rational function with simple poles at points in $z_{1}, z_{2}, \ldots, z_{N}$ in $[0,1]$ on the real axis. Milton $[7,8]$ correctly observes that this need not be so and cites examples to the contrary. However the calculation of bounds can be carried out correctly with only a rational form for $F_{i i}(s)$ as Milton notes. The mathematical reason for this will be seen in the next section.

2. From the symmetric formula (3.11) it follows that $m_{i k}(h)$ of (4.9) becomes

$$
m_{l k}(h)=\sum_{i=1}^{d} \int_{\Omega} P(d \omega)\left(\tilde{\chi}_{1}(\omega)+h \tilde{\chi}_{2}(\omega)\right) \widetilde{E}_{i}^{k} \widetilde{E}_{i}^{l} .
$$

From $\left(4.9^{\prime}\right)$ it follows that $\operatorname{Im} m_{i i}(h)>0$ when $\operatorname{Im} h>0$. Therefore the function $-F_{i i}(s)=m_{i i}(h)-1$ with $s$ given by (4.11) also has positive imaginary part when $\operatorname{Im} s>0$. Moreover there is a constant $M$ such that

$$
\left|s F_{i i}(\sqrt{-1} s)\right| \leqq M, \quad s>0
$$


since $m_{i i}(h)$ is analytic near $h=1$. But by a general representation theorem in function theory [19, Vol. II, p. 8] it follows that there exists a positive measure $\mu_{i i}(d z)$ on $(-\infty, \infty)$ such that

$$
-F_{i i}(s)=\int_{-\infty}^{\infty} \frac{\mu_{i i}(d z)}{z-s} .
$$

This is precisely (4.12) provided we use the additional information that $m_{i i}(h)$ is analytic everywhere except on the negative real axis in order to limit the support of $\mu_{i i}$ to the interval $[0,1]$. Thus we have proved (4.12) directly (at least when $i=k$ ) but it is instructive to prove it also using the spectral theorem as we do below. Of course, the spectral theorem itself is proved using the above function theoretic representation formula so the proofs are not all that different.

Proof. The formal equation (4.10), understood to hold in the variational form (3.7), can be rewritten as

$$
\sum_{i=1}^{d} L_{i} \widetilde{G}_{i}^{k}+(h-1) \sum_{i=1}^{d} L_{i}\left[\tilde{\chi}_{2}\left(\tilde{G}_{i}^{k}+\delta_{i k}\right)\right]=0 .
$$

Let us define the elliptic operator

$$
\tilde{\Delta}=\sum_{i=1}^{d} L_{i}^{2}
$$

and formally the operators

$$
\tilde{A}_{j i}=L_{j}(-\tilde{\Delta})^{-1} L_{i}, \quad i, j=1,2, \ldots, d .
$$

One can verify by spectral theory (the $L_{i}$ commute with each other) that the operators $\tilde{A}_{j i}$ are well defined and bounded in $L^{2}(\Omega, \mathscr{F}, P)$ and have norm less than or equal to one, just as in the usual case where $L_{i}=\partial / \partial x_{i}$.

Define the Hilbert space $\mathscr{K}$ by

$$
\mathscr{K}==\left\{\tilde{f}_{i}(\omega) \in L^{2}(\Omega, \mathscr{F}, P), \quad i=1,2, \ldots, d\right\},
$$

but now with the inner product

$$
\langle\tilde{f}, \tilde{g}\rangle=\int_{\Omega} P(d \omega) \tilde{\chi}_{2}(\omega) \sum_{i=1}^{d} \tilde{f}_{i}(\omega) \tilde{g}_{i}(\omega) .
$$

The operator $\widetilde{\mathscr{B}}$ on $\mathscr{K}$ defined by

$$
(\widetilde{B} \widetilde{f})_{j}(\omega)=\sum_{i=1}^{d} \tilde{A}_{j i}\left(\tilde{\chi}_{2} \tilde{f_{i}}\right)(\omega)
$$

is again bounded in norm by one and is selfadjoint under the inner product (4.17). Recall that the operators $L_{i}$ are skew adjoint.

We can rewrite problem (4.13) in terms of $\tilde{A}_{j i}$, hence in terms of $\widetilde{B}$, as follows

$$
\tilde{G}_{j}^{k}+\delta_{j k}+(1-h) \sum_{i=1}^{d} \tilde{A}_{j i}\left[\tilde{\chi}_{2}\left(\tilde{G}_{i}^{k}+\delta_{i k}\right)\right]=\delta_{j k}, \quad j, k=1,2, \ldots, d .
$$


The equivalence of (4.19) to (4.13) is seen by applying $L_{j}$ to (4.19) (in distribution sense) and summing on $j$. Recalling the notation (4.11), we may solve (4.19) when $s$ is outside $[-1,1]$, obtaining

$$
\tilde{G}_{j}^{k}+\delta_{j k}=s\left[(s+\widetilde{B})^{-1} e_{k}\right]_{j},
$$

since $\widetilde{B}$ is selfadjoint and $\|\widetilde{B}\|<1$. Here $e_{k}$ are the unit vectors with components $\left(\delta_{i k}\right)$. Moreover there exist projection-valued measures $Q(d z)$ on $[-1,1]$ such that

$$
\tilde{G}_{j}^{k}(\omega)+\delta_{j k}=s \int_{-1}^{1} \frac{\left(Q(d z) e_{k}\right)_{j}}{s+z}, \text { outside }[-1,1] .
$$

We know already from (4.7) that $\tilde{G}_{j}^{k}(\omega)+\delta_{j k}$ exists for all complex $h$ outside the negative real axis and hence by $(4.11)$ for all a outside $[0,1]$. This means that the integral in (4.21) runs only over $[-1,0]$. Therefore, after redefining the $Q(d z)$, we have

$$
\tilde{G}_{j}^{k}(\omega)+\delta_{j k}=s \int_{0}^{1} \frac{\left(Q(d z) e_{k}\right)_{j}}{s-z}, s \text { outside }[0,1] .
$$

From (4.9) we have that

$$
\begin{aligned}
m_{i k}(h) & =\delta_{i k}+(h-1) \int P(d \omega) \tilde{\chi}_{2}\left(\tilde{G}_{i}^{k}+\delta_{i k}\right) \\
& =\delta_{i k}-\frac{1}{s} \int P(d \omega) \tilde{\chi}_{2}\left(\tilde{G}_{i}^{k}+\delta_{i k}\right),
\end{aligned}
$$

or from (4.11)

$$
F_{i k}(s)=\frac{1}{s} \int P(d \omega) \tilde{\chi}_{2}\left(\tilde{G}_{i}^{k}+\delta_{i k}\right) .
$$

Now using (4.22) and recalling the form (4.17) of our inner product, we obtain

$$
F_{j k}(s)=\int_{0}^{1} \frac{\mu_{j k}(d z)}{s-z}, j, k=1,2, \ldots, d
$$

with

$$
\begin{aligned}
\mu_{j k}(d z) & =\int_{\Omega} P(d \omega) \tilde{\chi}_{2}(\omega)\left(Q(d z) e_{k}\right)_{j}(\omega) \\
& =\left\langle e_{j}, Q(d z) e_{k}\right\rangle .
\end{aligned}
$$

From this last statement the positivity of the diagonal measures $\mu_{i i}(d z)$ follows and the proof is complete.

By considering the problem for $D_{j}$ described briefly at the end of Sect. 3 (problem (2.3) $-(2.5)$ and $\left(2.6^{\prime}\right)$ ) for a two component medium, one arrives at the fact that the inverse matrix $\left(\varepsilon^{*}\right)_{i j}^{-1}(h)$ is analytic in $h$ except on the negative real axis. Hence $\left(m^{-1}\right)_{i j}(h)$ is analytic in $h$ except on the negative real axis. In the isotropic case where $m_{i j}(h)=\delta_{i j} m(h)$, this means that if $m(h)$ is meromorphic, which is not true in general, then all of its zeroes and poles lie on the negative real axis. 


\section{Perturbation Calculations about $\varepsilon_{1}=\varepsilon_{2}$ in a Two Component Medium and Their Analytic Continuation}

Perhaps the only case in which $\varepsilon_{i k}^{*}$ can be calculated rather simply, without special hypotheses about the form of $\varepsilon_{i j}(x, \omega)$, is when the $\varepsilon_{i j}$ is close to $\delta_{i j}$ uniformly in $x$ and $\omega$. Such calculations are reported in [13] and we reproduce a few here for the twocomponent case (4.1).

From (4.4) and (4.9) it suffices to calculate $m_{i k}(h)$ and its derivatives with respect to $h$ at $h=1$. Physically it is clear that

$$
m_{i k}(1)=\delta_{i k},
$$

which is also implied by (4.11) and (4.12) since $h \rightarrow 1$ corresponds to $s \rightarrow \infty$. Since $m_{i k}(h)$ is analytic, it has a power series expansion

$$
m_{i k}(h)=\delta_{i k}+\sum_{n=1}^{\infty} \frac{m_{i k}^{(n)}(1)}{n !}(h-1)^{n}, m_{i k}^{(n)}(h)=\left(\frac{d}{d h}\right)^{n} m_{i k}(h),
$$

which converges for $|h-1|<1$. Using (4.11) we may rewrite (5.2) in the form

$$
F_{i k}(s)=-\sum_{n=1}^{\infty} \frac{1}{n !} m_{i k}^{(n)}(1)\left(\frac{-1}{s}\right)^{n},|s|>1 .
$$

But from (4.12) we have for $|s|>1$,

where

$$
F_{i k}(s)=\sum_{n=1}^{\infty} \frac{1}{s^{n}} \mu_{i k}^{(n-1)},
$$

$$
\mu_{i k}^{(n-1)}=\int_{0}^{1} z^{n-1} \mu_{i k}(d z), \quad n=1,2, \ldots
$$

are the moments of the measure $\mu_{i k}(d z)$. Comparing (5.4) and (5.3) we see that

$$
\mu_{i k}^{(n-1)}=\frac{(-1)^{n-1}}{n !} m_{i k}^{(n)}(1), \quad n=1,2, \ldots, i, k=1,2, \ldots, d .
$$

When $i=k$ the moments of the positive measures are completely determined by (5.6). Since positive finite measures on a compact set are uniquely determined by their moments, it follows that:

The values of $(d / d h)^{n} m_{i i}(h)$ at $h=1$ completely

determine $m_{i i}(h)$ everywhere in the complex $h$

plane except on the negative real axis.

The conclusion (5.7) shows the usefulness of the representation (4.12) in converting local information at $h=1$ to global information.

Now we calculate $m_{i k}^{(1)}(h)$ at $h=1$. Differentiating (4.9) we have

$$
\begin{aligned}
\left.\frac{d}{d h} m_{i k}(h)\right|_{h=1} & =\int_{\Omega} P(d \omega)\left[\tilde{\chi}_{2}(\omega) \tilde{E}_{i}^{k}(\omega)+\left(\tilde{\chi}_{1}(\omega)+h \tilde{\chi}_{2}(\omega)\right) \frac{d}{d h} \tilde{E}_{i}^{k}(\omega)\right]_{h=1} \\
& =\int_{\Omega} P(d \omega) \tilde{\chi}_{2}(\omega) \delta_{i k}=p_{2} \delta_{i k},
\end{aligned}
$$


where $p_{2}$ is the volume fraction occupied by the dielectric material of type two and is defined by the last equality. Using (5.8) in (5.6) we obtain

$$
\mu_{i k}^{(0)}=\int_{0}^{1} \mu_{i k}(d z)=p_{2} \delta_{i k} .
$$

To compute $m_{i k}^{(2)}(1)$ we differentiate (5.8) again and obtain

$$
\begin{aligned}
\frac{d^{2}}{d h^{2}} m_{i k}(h) & =\int_{\Omega} P(d \omega)\left[2 \tilde{\chi}_{2}(\omega) \frac{d}{d h} \tilde{E}_{i}^{k}(\omega)+\left(\tilde{\chi}_{1}+h \tilde{\chi}_{2}\right) \frac{d^{2}}{d h^{2}} \tilde{E}_{i}^{k}\right]_{h=1} \\
& =\left.2 \int_{\Omega} P(d \omega) \tilde{\chi}_{2}(\omega) \frac{d}{d h} \tilde{E}_{i}^{k}\right|_{h=1} .
\end{aligned}
$$

We must now calculate $\left.(d / d h) \tilde{E}_{i}^{k}\right|_{h=1}$. We differentiate (4.10) with respect to $h$, evaluate at $h=1$ and get

$$
L_{i}\left(\left.\frac{d}{d h} \tilde{E}_{i}^{k}\right|_{h=1}+\tilde{\chi}_{2} \delta_{i k}\right)=0 .
$$

Thus, in terms of the operators $\tilde{A}_{j i}$ of (4.15),

$$
\left.\frac{d}{d h} \tilde{E}_{j}^{k}\right|_{h=1}=\sum_{j=1}^{d} \tilde{A}_{j i}\left(\tilde{\chi}_{2} \delta_{i k}\right)=\tilde{A}_{j k}\left(\tilde{\chi}_{2}\right) .
$$

Using (5.11) in (5.8) yields

$$
\left.\frac{d^{2}}{d h^{2}} m_{i k}(h)\right|_{h=1}=2 \int_{\Omega} P(d \omega) \tilde{\chi}_{2}(\omega)\left(\tilde{A}_{j k} \tilde{\chi}_{2}\right)(\omega) .
$$

To evaluate the integral on the right of (5.12) we note the following. The boundedness of the operators $\tilde{A}_{j k}$ in $L^{2}(\Omega, \mathscr{F}, P)$ implies that the differential operators

$$
A_{j i}=\frac{\partial}{\partial x_{j}}(-\Delta)^{-1} \frac{\partial}{\partial x_{i}}, \quad i, j=1,2, \ldots, d,
$$

which are bounded on $L^{2}\left(\mathbb{R}^{d}\right)$, are also bounded operators on square integrable stationary random fields. Thus we may write (5.12) in the form

$$
\begin{aligned}
\left.\frac{d^{2}}{d h^{2}} m_{i k}(h)\right|_{h=1} & =2 \int_{\Omega} P(d \omega) \chi_{2}(x, \omega) \frac{\partial^{2}}{\partial x_{i} \partial x_{k}}(-\Delta)^{-1}\left(\chi_{2}(x, \omega)-p_{2}\right) \\
& =\int_{R^{d}} \Gamma_{i k}(y) R(y) d y
\end{aligned}
$$

where $\Gamma_{i k}(y)$ is the kernel of the singular integral operator $A_{i k}$ of $(5.13)$ and $R(y)$ is the correlation function

$$
R(y)=\int P(d \omega) \chi_{2}(x+y, \omega)\left(\chi_{2}(x, \omega)-p_{2}\right) .
$$

If $R(y)$ depends on $|y|$ only, the case of statistically isotropic random geometry, then

$$
\left.\frac{d^{2}}{d h^{2}} m_{i k}(h)\right|_{h=1}=-\frac{2}{d} R(0) \delta_{i k}=-\frac{2 p_{2} p_{1}}{d} \delta_{i k},
$$


where $p_{1}=1-p_{2}$ is the volume fraction of dielectric material of type one.

Thus from (5.6) and (5.16) we have in the isotropic random geometry case

$$
\mu_{i k}^{(1)}=\frac{1}{d} p_{1} p_{2} \delta_{i k}
$$

In the anisotropic case $\mu_{i k}^{(1)}$ depends on the full correlation function $R(y)$ and not just its value at zero. Moreover, the higher moments $\mu_{i k}^{(n)}, n \geqq 2$ depend on $(n+1)$-point correlation functions in general. A formula can be derived for them, in the same way as above, with the use of the kernel $\Gamma_{i k}(y)$ and its iterates.

\section{Range of Values of the Effective Coefficient}

We shall now fix attention at one diagonal coefficient $m_{11}(h)$ and call it $m(h)$. We rewrite (4.11) and (4.12) in this case as

$$
\begin{aligned}
1-m(h)=F(s) & =\int_{0}^{1} \frac{\mu(d z)}{s-z}, s \text { outside }[0,1], \\
s & =\frac{1}{1-h} .
\end{aligned}
$$

Evidently $F(s)$ depends also on $\mu$ which belongs to $\mathscr{M}$, the set of positive finite Borel measures on $[0,1]$. We shall therefore write $F(s, \mu)$.

For $s$ fixed outside the unit interval on the real axis, $F$ is a linear mapping of $\mathscr{M}$ to the complex plane as can be seen from (6.1). Let

$$
\mathscr{M}\left(\mu^{(0)}, \mu^{(1)}, \ldots, \mu^{(N)}\right)=\left\{\mu \in \mathscr{M} \mid \int_{0}^{1} z^{n} \mu(d z)=\mu^{(n)}, n=0,1, \ldots, N\right\},
$$

provided that the numbers $\mu^{(0)}, \mu^{(1)}, \ldots, \mu^{(N)}$ form a positive definite sequence [20] so that they can represent the moments of a measure. From (6.1) we see that for fixed $s$, $F(s, \mu)$ is a linear mapping of $\mathscr{M}\left(\mu^{(0)}, \ldots, \mu^{(N)}\right)$ to the complex plane $\mathbb{C}$. We denote by

$$
\Lambda\left(h, \mu^{(0)}, \ldots, \mu^{(N)}\right)=\left\{1-F(s, \mu) \in \mathbb{C} \mid \operatorname{Re} s \notin[0,1], \mu \in \mathscr{M}\left(\mu^{(0)}, \ldots, \mu^{(N)}\right)\right\} .
$$

we call the subset $\Lambda$ of $\mathbb{C}$ the range of values of the effective (relative) dielectric constant $m(h)$ when $h$ is given along with $\mu^{(0)}, \mu^{(1)}, \ldots, \mu^{(N)}$ and $\mu$ varies over all measures with given first $N$ moments. The problem is to characterize this set because, as pointed out at the end of Sect. 5 , if we have information about the first $N+1$ correlation functions of the random medium then we can determine the first $N$ moments of $\mu$. The set $\Lambda$ represents the possible values of $m(h)$ compatible with this information about the medium.

Actually, $\Lambda$ is not the precise range of the values of $m(h)$ as our terminology suggests because although each random geometry gives rise to a measure $\mu$ in the representation (6.1), we know that not every measure $\mu$ in (6.1) gives a function $m(h)$ that is the effective (relative) dielectric constant of a random medium. For example, in dimension $d=2$ Keller [21] showed that

$$
m(h) m\left(\frac{1}{h}\right)=1
$$


and Schulgasser [22] showed that for $d \geqq 3$ and $h \geqq 1$

$$
m(h) m\left(\frac{1}{h}\right) \geqq 1 \text {. }
$$

Both of these statements are proved in [14] in a very simple manner. From (6.4) or (6.5) we see that not every measure $\mu$ in (6.1) comes from a random geometry. Therefore the set $\Lambda$ will in general contain the exact range of values of the effective dielectric constant. For simplicity, we shall continue to refer to $\Lambda$ as the range of values.

The set of measures $\mathscr{M}\left(\mu^{(0)}, \mu^{(1)}, \ldots, \mu^{(N)}\right)$ is a compact, convex subset of $\mathscr{M}$ with the topology of weak convergence. Since the mapping (6.1) is linear in $\mu$ it follows that the range of values $\Lambda$ of (6.3) is a compact convex subset of the complex plane $\mathbb{C}$. The extreme points of the set $\mathscr{M}\left(\mu^{(0)}\right)$ are the one point measures $\alpha \delta_{y}(d z), 0 \leqq y<1$, $\alpha \geqq 0$, since they cannot be expressed as convex combinations of other measures while all other measures are convex combinations of one point measures. One can verify [23] that the extreme points of $\mathscr{M}\left(\mu^{(0)}, \mu^{(1)}, \ldots, \mu^{(N)}\right)$ are weak limits of convex combinations of $(N+1)$-point measures, i.e. measures of the form

$$
\mu(d z)=\sum_{k=1}^{N+1} \alpha_{k} \delta_{z_{k}}(d z)
$$

where

$$
\alpha_{k} \geqq 0,0 \leqq z_{1}<z_{2}<\ldots<z_{N+1}<1, \sum_{k=1}^{N+1} \alpha_{k} z_{k}^{n}=\mu^{(n)}, n=0,1, \ldots, N .
$$

We conclude from the above that in order to determine the extreme points of $\Lambda\left(h, \mu^{(0)}, \ldots, \mu^{(N)}\right)$ it suffices to determine the range of values in $\mathbb{C}$ of

$$
m(h)=1-\sum_{k=1}^{N+1} \frac{\alpha_{k}}{s-z_{k}}, \quad s=\frac{1}{1-h},
$$

as $\alpha_{k}$ and $z_{k}$ vary while (6.7) holds. This is exactly the point at which Bergman and Milton begin their analyses, by postulating essentially that $m(h)$ should have the form (6.8), perhaps in some approximate sense. We see that actually no approximations are involved. While (6.8) may not run over all points in $\Lambda$, it certainly runs over all extreme points of $\Lambda$, which is sufficient to characterize this set since it is convex.

In the remainder of this section we shall consider some consequences of (6.8). Let $\zeta_{1}, \zeta_{2}, \ldots, \zeta_{N+1}$ be the zeroes of $m(h)$ which are necessarily real. Then we can write

$$
\prod_{j=1}^{N+1} \frac{s-\zeta_{j}}{s-z_{j}}=1-\sum_{j=1}^{N+1} \frac{\alpha_{j}}{s-z_{j}}
$$

and hence

$$
0 \leqq \alpha_{p}=-\frac{\prod_{j=1}^{N+1}\left(z_{p}-\zeta_{j}\right)}{\prod_{j \neq p}\left(z_{p}-z_{j}\right)}, p=1,2, \ldots, N+1
$$


This implies that

$$
0 \leqq z_{N+1} \leqq \zeta_{N+1} \leqq \ldots \leqq \zeta_{2} \leqq z_{1} \leqq \zeta_{1} \leqq 1 .
$$

Let us consider the form of the set $\Lambda\left(h, \mu^{(0)}\right)$, where by (5.9), $\mu^{(0)}=p_{2}$. We have

$$
\Lambda\left(h, \mu^{(0)}\right)=\left\{m=1-\frac{p_{2}}{s-z}, 0 \leqq z \leqq 1-p_{2}\right\} .
$$

When $h$ is real and bigger than one, then $s$ is negative. In this case one sees easily that $\Lambda\left(h, \mu^{(0)}\right)$ is the interval

$$
1-\frac{p_{2}}{s-\left(1-p_{2}\right)} \leqq m \leqq 1-\frac{p_{2}}{s}
$$

which is equivalent to

$$
\frac{1}{1-p_{2}+\frac{p_{2}}{h}} \leqq m \leqq 1-p_{2}+h p_{2} .
$$

These are the classical arithmetic and harmonic mean bounds for the effective dielectric constant of a two-component dielectric material. They are achieved by plane parallel configurations of the material. When $h$ is complex, the convex hull of the set $\Lambda\left(h, \mu^{(0)}\right)$ is bounded by a circular arc and a cord.

One can describe also the set $\Lambda\left(h, \mu^{(0)}, \mu^{(1)}\right)$ in the isotropic case when $\mu^{(0)}=p_{2}$ and $\mu^{(1)}=p_{1} p_{2} / d$ by (5.9) and (5.17). When $h$ is real and bigger than one, the Hashin Shtrikman inequalities are obtained

$$
1+\frac{p_{2}}{\frac{1}{h-1}+\frac{p_{1}}{d}} \leqq m(h) \leqq h+\frac{p_{1}}{\frac{1}{1-h}+\frac{p_{2}}{d h}} .
$$

In the complex case the convex hull is bounded again by circular arcs.

\section{Appendix. Existence of Effective Dielectric Constant}

In Sect. 2 we pointed out that the definition of effective dielectric constant via (2.3)(2.7) (or more precisely (3.7)-(3.10)) is consistent with the more usual definition where a finite volume of material is taken at first and then $\varepsilon_{i j}^{*}$ is obtained in an infinite volume limit. We shall analyze in detail in this appendix this limit. For simplicity we shall consider the two-dimensional isotropic case; the general case goes through the same way. The analysis follows [18] suitably modified to the present case.

Let the material with dielectric constant $\varepsilon\left(x_{1}, x_{2}, \omega\right)$ such that $0<\alpha \leqq$ $\varepsilon\left(x_{1}, x_{2}, \omega\right) \leqq \beta<\infty$ occupy the square $-N \leqq x_{j} \leqq N, j=1,2$. Let $\tilde{u}^{(N)}\left(x_{1}, x_{2}, \omega\right)$ be the solution of

$$
\begin{gathered}
\nabla \cdot\left(\varepsilon \nabla \tilde{u}^{(N)}\right)=0,-N<x_{j}<N, \quad j=1,2, \\
\frac{\partial \tilde{u}^{(N)}}{\partial x_{2}}=0 \text { on } x_{2}=N \text { or } x_{2}=-N,-N \leqq x_{1} \leqq N,
\end{gathered}
$$




$$
\begin{aligned}
\tilde{u}^{(N)} & =-N \text { on } x_{1}=-N,-N \leqq x_{2} \leqq N, \\
\tilde{u}^{(N)} & =+N \text { on } x_{1}=+N,-N \leqq x_{2} \leqq N . \\
\varepsilon^{*, N}(\omega) & =\frac{1}{2 N} \int_{-N}^{N} \varepsilon\left(x_{1}, x_{2}, \omega\right) \frac{\partial \tilde{u}^{(N)}\left(x_{1}, x_{2}, \omega\right)}{\partial x_{1}} d x_{2} .
\end{aligned}
$$

We note that this quantity is independent of $x_{1}$ in view of (A.1).

We introduce also the problem (2.3)-(2.6) and (2.7) suitably specialized in $\mathbb{R}^{2}$ with isotropic $\varepsilon_{i j}$.

$$
\begin{aligned}
& D(x, \omega)=\varepsilon(x, \omega) E(x, \omega), x=\left(x_{1}, x_{2}\right), \\
& \nabla \times E=0, \\
& \nabla \cdot D=0, \\
& \int_{\Omega} P(d \omega) E_{j}(x, \omega)=\delta_{1 j}, j=1,2, \\
& \varepsilon^{*}=\int_{\Omega} P(d \omega) D_{1}(x, \omega) .
\end{aligned}
$$

of course here $E$ and $D$ are stationary fields and (A.6)-(A.9) is taken in variational form as in Sect. 3.

We shall show that, when $\varepsilon(x, \omega)$ is stationary and ergodic then

$$
\int_{\Omega} P(d \omega)\left(\varepsilon^{*, N}(\omega)-\varepsilon^{*}\right)^{2} \rightarrow 0, \text { as } N \rightarrow \infty,
$$

which means that the average dielectric displacement over a vertical line for a square of material of side $2 N$ tends to the effective dielectric constant $\varepsilon^{*}$ as $N \rightarrow \infty$ in mean square.

To prove (A.11) we first transform (A.1)-(A.4) as follows. Let

$$
u^{(N)}\left(x_{1}, x_{2}, \omega\right)=\frac{1}{N} \tilde{u}^{(N)}\left(N x_{1}, N x_{2}, \omega\right) .
$$

Then $u^{(N)}\left(x_{1}, x_{2}, \omega\right)$ is defined in $\left|x_{1}\right| \leqq 1,\left|x_{2}\right| \leqq 1$ and we have

$$
\begin{gathered}
\nabla \cdot\left(\varepsilon\left(N x_{1}, N x_{2}, \omega\right) \nabla u^{(N)}\left(x_{1}, x_{2}, \omega\right)\right)=0,\left|x_{1}\right|<1,\left|x_{2}\right|<1, \\
\left.\frac{\partial u^{(N)}}{\partial x_{2}}\left(x_{1}, x_{2}, \omega\right)\right|_{x_{2}= \pm 1}=0,\left|x_{1}\right| \leqq 1 \\
u^{(N)}\left( \pm 1, x_{2}, \omega\right)= \pm 1,\left|x_{2}\right| \leqq 1
\end{gathered}
$$

Now let

$$
\chi\left(x_{1}, x_{2}, \omega\right)=\int_{0}^{|x|}\left[\frac{x_{1}}{|x|} G_{1}\left(\frac{s x_{1}}{|x|}, \frac{s x_{2}}{|x|}, \omega\right)+\frac{x_{2}}{|x|} G_{2}\left(\frac{s x_{1}}{|x|}, \frac{s x_{2}}{|x|}, \omega\right)\right] d s,
$$

where the stationary field $E$ of (A.6)-(A.9) is written in the form

$$
E_{j}(x, \omega)=G_{j}(x, \omega)+\delta_{1 j}, j=1,2, \int_{\Omega} P(d \omega) G_{j}(x, \omega)=0 .
$$


It is easily seen that because of (A.7) we have that

$$
G(x, \omega)=\nabla \chi(x, \omega) \text {. }
$$

Moreover, since $\varepsilon(x, \omega)$ is ergodic, so is $G(x, \omega)$, and thus

$$
\int_{\Omega} P(d \omega)\left(\frac{1}{|x|} \chi(x, \omega)\right)^{2} \rightarrow 0 \text { as }|x| \rightarrow \infty,
$$

because $G$ in (A.16) has mean zero. Let

$$
\chi^{(N)}\left(x_{1}, x_{2}, \omega\right)=\frac{1}{N} \chi\left(N x_{1}, N x_{2}, \omega\right)
$$

We want to prove (A.11). First we note that since the process $\varepsilon(x, \omega)$ is ergodic

$$
\frac{1}{(2 N)^{2}} \int_{-N}^{N} \int_{-N}^{N} d x_{1} d x_{2} \varepsilon\left(x_{1}, x_{2}, \omega\right)\left(1+\frac{\partial \chi\left(x_{1}, x_{2}, \omega\right)}{\partial x_{1}}\right) \rightarrow \varepsilon^{*}
$$

as $N \rightarrow \infty$ in mean square (or with probability one), and hence

$$
\frac{1}{4} \int_{-1}^{1} \int_{-1}^{1} d x_{1} d x_{2} \varepsilon^{(N)}\left(x_{1}, x_{2}, \omega\right) \frac{\partial}{\partial x_{1}}\left(x_{1}+\chi^{(N)}\left(x_{1}, x_{2}, \omega\right)\right) \rightarrow \varepsilon^{*}
$$

as $N \rightarrow \infty$, where

$$
\varepsilon^{(N)}\left(x_{1}, x_{2}, \omega\right)=\varepsilon\left(N x_{1}, N x_{2}, \omega\right)
$$

In view of (A.21) or (A.21') it suffices for (A.11) to prove

$$
\begin{aligned}
0= & \lim _{N \rightarrow \infty} \int_{\Omega} P(d \omega)\left\{\frac { 1 } { 4 } \int _ { - 1 } ^ { 1 } \int _ { - 1 } ^ { 1 } d x _ { 1 } d x _ { 2 } \varepsilon ^ { ( N ) } ( x _ { 1 } , x _ { 2 } , \omega ) \left[\frac{\partial u^{(N)}\left(x_{1}, x_{2}, \omega\right)}{\partial x_{1}}\right.\right. \\
& \left.-\frac{\partial}{\partial x_{1}}\left(x_{1}+\chi^{(N)}\left(x_{1}, x_{2}, \omega\right)\right]\right\}^{2} .
\end{aligned}
$$

But

$$
\begin{aligned}
& \int_{\Omega} P(d \omega)\left\{\frac{1}{4} \int_{-1}^{1} \int_{-1}^{1} d x_{1} d x_{2} \varepsilon^{(N)}\left(x_{1}, x_{2}, \omega\right)\left[\frac{\partial u^{(N)}}{\partial x_{1}}-\frac{\partial}{\partial x_{1}}\left(x_{1}+\chi^{(N)}\right)\right]\right\}^{2} \\
& \leqq \beta^{2} \int_{\Omega} P(d \omega) \frac{1}{4} \int_{-1}^{1} \int_{-1}^{1} d x_{1} d x_{2}\left|\nabla z^{(N)}\left(x_{1}, x_{2}, \omega\right)\right|^{2}
\end{aligned}
$$

where we have used the fact that $\alpha \leqq \varepsilon \leqq \beta<\infty$, Schwarz's inequality and

$$
z^{(N)}\left(x_{1}, x_{2}, \omega\right)=u^{(N)}\left(x_{1}, x_{2}, \omega\right)-\left(x_{1}+\chi^{(N)}\left(x_{1}, x_{2}, \omega\right)\right) .
$$

Let $\tau(N)$ be a function of $N$ tending to zero as $N \rightarrow \infty$ in a manner that will be made precise later. Let $\gamma(s), s \geqq 0$ be a $C^{\infty}$ function such that

$$
\gamma(s)=\left\{\begin{array}{l}
1, s \geqq 2 \\
0, s \leqq 1
\end{array}\right.
$$


and put

$$
\psi^{(N)}\left(x_{1}, x_{2}\right)=\gamma\left(\frac{1-x_{1}}{\tau(N)}\right) \gamma\left(\frac{1+x_{1}}{\tau(N)}\right) \gamma\left(\frac{1-x_{2}}{\tau(N)}\right) \gamma\left(\frac{1+x_{2}}{\tau(N)}\right),
$$

so that $\psi^{(N)}$ vanishes identically near the boundary of $\left|x_{1}\right|<1,\left|x_{2}\right|<1$. Let

so that

$$
\begin{aligned}
& \hat{z}^{(N)}\left(x_{1}, x_{2}, \omega\right)=u^{(N)}\left(x_{1}, x_{2}, \omega\right)-x_{1}-\hat{\chi}^{(N)}\left(x_{1}, x_{2}, \omega\right) \\
& \hat{\chi}^{(N)}\left(x_{1}, x_{2}, \omega\right)=\chi^{(N)}\left(x_{1}, x_{2}, \omega\right) \psi^{(N)}\left(x_{1}, x_{2}\right)
\end{aligned}
$$

$$
\hat{z}^{(N)}=z^{(N)}+\chi^{(N)}\left(1-\psi^{(N)}\right) .
$$

From (A.13), (A.17), (A.18), (A.6), (A.8) and (A.29) it follows that

$$
\begin{gathered}
\nabla \cdot\left(\varepsilon^{(N)} \nabla \hat{z}^{(N)}\right)=\nabla \cdot\left[\varepsilon^{(N)} \nabla\left(\chi^{(N)}\left(1-\psi^{(N)}\right)\right)\right], \\
\frac{\partial \hat{z}^{(N)}}{\partial x_{2}}=0 \text { on } x_{2}= \pm 1,\left|x_{1}\right| \leqq 1, \\
\hat{z}^{(N)}=0 \text { on } x_{1}= \pm 1,\left|x_{2}\right| \leqq 1 .
\end{gathered}
$$

We shall now transform the right side of (A.30) suitably. Let $e_{1}$ and $e_{2}$ be unit vectors in the direction $x_{1}$ and $x_{2}$ respectively. From the definition of $\chi$ it follows that

Moreover from (A.10)

$$
\nabla \cdot\left[\varepsilon^{(N)}\left(\nabla \chi^{(N)}+e_{1}\right)\right]=0 .
$$

$$
\varepsilon^{*}=\int_{\Omega}\left[\varepsilon^{(N)}\left(\nabla \chi^{(N)}+e_{1}\right) \cdot e_{1}\right] P(d \omega) .
$$

We have assumed, without loss of generality, that the effective dielectric tensor $\varepsilon_{i j}^{*}$ is isotropic $\varepsilon_{i j}^{*}=\delta_{i j} \varepsilon^{*}$. This implies that

$$
0=\int_{\Omega}\left[\varepsilon^{(N)}\left(\nabla \chi^{(N)}+e_{1}\right) \cdot e_{2}\right] P(d \omega) .
$$

With these observations in mind we rewrite the right side of (A.30).

$$
\begin{aligned}
\nabla & \cdot\left[\varepsilon^{(N)} \nabla\left(\chi^{(N)}\left(1-\psi^{(N)}\right)\right)\right] \\
& =\nabla \cdot\left[\varepsilon^{(N)} \nabla \chi^{(N)}\right]\left(1-\psi^{(N)}\right)-\varepsilon^{(N)} \nabla \chi^{(N)} \cdot \nabla \psi^{(N)}-\nabla \cdot\left[\varepsilon^{(N)} \chi^{(N)} \nabla \psi^{(N)}\right] \\
& =-\nabla \cdot\left[\varepsilon^{(N)} e_{1}\right]\left(1-\psi^{(N)}\right)-\varepsilon^{(N)} \nabla \chi^{(N)} \cdot \nabla \psi^{(N)}-\nabla \cdot\left[\varepsilon^{(N)} \chi^{(N)} \nabla \psi^{(N)}\right] .
\end{aligned}
$$

Now we multiply (A.30) by $\hat{z}^{(N)}$, integrate both sides over $\left|x_{1}\right| \leqq 1,\left|x_{2}\right| \leqq 1$ and integrate by parts. Because of (A.31) and (A.32) and the fact that $\psi^{(N)}$ vanishes identically near the boundary of the square, there are no boundary contributions. Thus

$$
\begin{aligned}
& \int_{-1}^{1} \int_{-1}^{1} \varepsilon^{(N)}\left(\nabla \hat{z}^{(N)}\right)^{2} d x_{1} d x_{2}=\int_{-1}^{1} \int_{-1}^{1}\left(\varepsilon^{*}-\varepsilon^{(N)}\right) \nabla \hat{z}^{(N)} \cdot e_{1}\left(1-\psi^{(N)}\right) d x_{1} d x_{2} \\
& +\int_{-1}^{1} \int_{-1}^{1}\left(\varepsilon^{(N)}\left(\nabla \chi^{(N)}+e_{1}\right)-\varepsilon^{*} e_{1}\right) \cdot \nabla \psi^{(N)} \hat{z}^{(N)} d x_{1} d x_{2} \\
& -\int_{-1}^{1} \int_{-1}^{1} \varepsilon^{(N)} \nabla \hat{z}^{(N)} \cdot \nabla \psi^{(N)} \chi^{(N)} d x_{1} d x_{2} .
\end{aligned}
$$


We note that because of (A.34) and (A.35)

$$
\int\left[\varepsilon^{(N)}\left(\nabla \chi^{(N)}+e_{1}\right)-\varepsilon^{*} e_{1}\right] P(d \omega)=0 .
$$

From the right side of the inequality in (A.24) it suffices to prove that

$$
I^{(N)}=\int_{\Omega} P(d \omega) \int_{-1}^{1} \int_{-1}^{1} d x_{1} d x_{2}\left(\nabla z^{(N)}\right)^{2} \rightarrow 0 \text { as } N \rightarrow \infty .
$$

But from (A.29)

$$
\begin{aligned}
I^{(N)} \leqq & 2 \int_{\Omega} P(d \omega) \int_{-1}^{1} \int_{-1}^{1} d x_{1} d x_{2}\left(\nabla \hat{z}^{(N)}\right)^{2} \\
& +2 \int_{\Omega} P(d \omega) \int_{-1}^{1} \int_{-1}^{1} d x_{1} d x_{2}\left(\nabla\left[\chi^{(N)}\left(1-\psi^{(N)}\right)\right]\right)^{2} \\
= & \hat{I}^{(N)}+J^{(N)}
\end{aligned}
$$

From (A.37) and the uniform upper and lower bounds for $\varepsilon(x, \omega)$ we see that we have to get a bound for $\hat{I}^{(N)}$ in terms of objects from the right side of (A.37). To proceed further we must rewrite the middle term on the right side of (A.37) as follows.

Let

$$
\begin{aligned}
& h_{1}\left(x_{1}, x_{2}, \omega\right)=\varepsilon\left(x_{1}, x_{2}, \omega\right)\left(\nabla \chi\left(x_{1}, x_{2}, \omega\right)+e_{1}\right) \cdot e_{1}-\varepsilon^{*}, \\
& h_{2}\left(x_{1}, x_{2}, \omega\right)=\varepsilon\left(x_{1}, x_{2}, \omega\right)\left(\nabla \chi\left(x_{1}, x_{2}, \omega\right)+e_{1}\right) \cdot e_{2} .
\end{aligned}
$$

These fields are stationary and have mean zero by (A.34) and (A.35). By a process similar to the one that goes from $G$ to $\chi$ (cf.(A.16), (A.18)) we can find vector fields $H_{1}$ and $\mathrm{H}_{2}$ such that

$$
\nabla \cdot H_{1}=h_{1}, \nabla \cdot H_{2}=h_{2} .
$$

These fields are not stationary but can be normalized to be zero at $\left(x_{1}, x_{2}\right)=0$ and

$$
\int_{\Omega} P(d \omega) \frac{1}{|x|}\left|H_{j}(x, \omega)\right|^{2} \rightarrow 0 \text { as }|x| \rightarrow \infty, j=1,2 .
$$

Defining

$$
\begin{aligned}
& H_{j}^{(N)}\left(x_{1}, x_{2}, \omega\right)=\frac{1}{N} H_{j}\left(N x_{1}, N x_{2}, \omega\right), j=1,2, \\
& h_{j}^{(N)}\left(x_{1}, x_{2}, \omega\right)=\frac{1}{N} h_{j}\left(N x_{1}, N x_{2}, \omega\right),
\end{aligned}
$$

we see that

$$
\varepsilon^{(N)}\left(\nabla \chi^{(N)}+e_{1}\right)-\varepsilon^{*} e_{1}=h_{1}^{(N)} e_{1}+h_{2}^{(N)} e_{2}=\left(\nabla \cdot H_{1}^{(N)}\right) e_{1}+\left(\nabla \cdot H_{2}^{(N)}\right) e_{2} .
$$

Thus,

$$
\int_{-1}^{1} \int_{-1}^{1}\left(\varepsilon^{(N)}\left(\nabla \chi^{(N)}+e_{1}\right)-\varepsilon^{*} e_{1}\right) \cdot \nabla \psi^{(N)} \hat{z}^{(N)} d x_{1} d x_{2}
$$




$$
\begin{aligned}
& =\int_{-1}^{1} \int_{-1}^{1}\left[\left(\nabla \cdot H_{1}^{(N)}\right) e_{1}+\left(\nabla \cdot H_{2}^{(N)}\right) e_{2}\right] \cdot \nabla \psi^{(N)} \hat{z}^{(N)} d x_{1} d x_{2} \\
& =-\int_{-1}^{1} \int_{-1}^{1}\left[H_{1}^{(N)} \cdot \nabla\left(e_{1} \cdot \nabla \psi^{(N)} \hat{z}^{(N)}\right)+H_{2}^{(N)} \cdot \nabla\left(e_{2} \cdot \nabla \psi^{(N)} \hat{z}^{(N)}\right)\right] d x_{1} d x_{2} .
\end{aligned}
$$

We now return to (A.37) and notice that when (A.45) is used to rewrite the middle term on the right and then Schwartz's inequality is used, the result $\widehat{I}^{(N)} \rightarrow 0$ (cf.(A.40)) will follow from Poincaré's inequality for $\hat{z}^{(N)}$ and

$$
\begin{gathered}
\int_{-1}^{1} \int_{-1}^{1}\left(1-\psi^{(N)}\right)^{2} d x_{1} d x_{2} \rightarrow 0, \\
\int_{\Omega} P(d \omega) \int_{-1}^{1} \int_{-1}^{1} d x_{1} d x_{2}\left|H_{j}^{(N)}\right|^{2}\left|\nabla\left(e_{1} \cdot \nabla \psi^{(N)}\right)\right|^{2} \rightarrow 0, j=1,2, \\
\int_{\Omega} P(d \omega) \int_{-1}^{1} \int_{-1}^{1} d x_{1} d x_{2}\left|H_{j}^{(N)}\right|^{2}\left|e_{1} \cdot \nabla \psi^{(N)}\right|^{2} \rightarrow 0, j=1,2, \\
\int_{\Omega} P(d \omega) \int_{-1}^{1} \int_{-1}^{1} d x_{1} d x_{2}\left(\chi^{(N)}\right)^{2}\left(\nabla \psi^{(N)}\right)^{2} \rightarrow 0, \text { as } N \rightarrow \infty
\end{gathered}
$$

We note in addition that $J^{(N)}$ in (A.40) will also go to zero provided (A.46) and (A.49) hold. Let

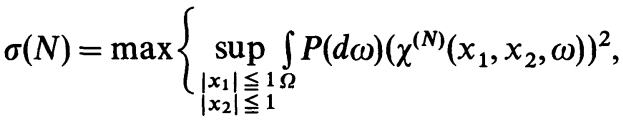

$$
\begin{aligned}
& \sup \int P(d \omega)\left|H_{1}^{(N)}\left(x_{1}, x_{2}, \omega\right)\right|^{2} \text {, } \\
& \left|\begin{array}{l}
x_{1} \mid \leqq 1 \\
\left|x_{2}\right| \leqq 1
\end{array}\right|
\end{aligned}
$$

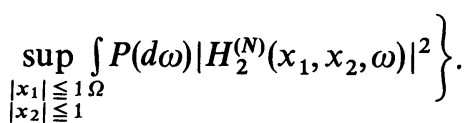

From (A.19) and (A.43) (slightly extended), it follows that $\sigma(N) \rightarrow 0$ as $N \rightarrow \infty$. Now since $1-\psi^{(N)}$ is zero except in a strip of width $2 \tau(N)$ near the boundary of the square and from (A.29),

$$
\begin{aligned}
\int_{-1}^{1} \int_{-1}^{1}\left(1-\psi^{(N)}\right)^{2} & \leqq \mathrm{C}_{1} \tau(N), \\
\left(\nabla \psi^{(N)}\right)^{2} & \leqq \frac{C_{2}}{(\tau(N))^{2}}, \\
\left(\nabla \nabla \psi^{(N)}\right)^{2} & \leqq \frac{C_{3}}{(\tau(N))^{4}},
\end{aligned}
$$

where $\left(\nabla \nabla \psi^{(N)}\right)^{2}$ stands for the sum of the squares of all second derivatives of $\psi^{(N)}$. With these facts (A.46)-(A.49) will follow provided

$$
\tau(N) \rightarrow 0, \frac{\sigma(N)}{(\tau(N))^{4}} \rightarrow 0, \frac{\sigma(N)}{(\tau(N))^{2}} \rightarrow 0 .
$$


We now choose $\tau(N)=(\sigma(N))^{1 / 8}$, for example, so that (A.52) clearly holds. This completes the proof of the theorem.

\section{References}

1 Bergman, D J : The dielectric constant of a composite material-a problem in classical physics Phys Rep C43, 377-407 (1978)

2 Bergman, D J : The dielectric constant of a simple cubic array of identical spheres J Phys C12, 4947-4960 (1979)

3 Bergman, D J , Stroud, D : Theory of resonances in electromagnetic scattering by macroscopic bodies Phys Rev B22, 3527 (1980)

4 Bergman, D J : Exactly solvable microscopic geometries and rigorous bounds for the complex dielectric constant of a two-component composite material Phys Rev Lett. 44, 1285-1287 (1980)

5 Bergman, D J : Bounds for the complex dielectric constant of a two-component composite material Phys Rev B23, 3058-3065 (1981)

6 Bergman, D J : Resonances in the bulk properties of composite media-Theory and applications In: Lecture Notes in Physics Vol 154 Berlin, Heidelberg, New York: Springer 1982, pp 10-37

7 Milton, G W : Bounds on the transport and optical properties of a two-component composite material J Appl Phys 52, 5294-304 (1981)

8 Milton, G W : Bounds on the complex permittivity of a two-component composite material $\mathrm{J}$ Appl Phys 52, 5286-93 (1981)

9 Milton, G W : Bounds on the electromagnetic, elastic and other properties of two-component composites Phys Rev Lett 46, 542-545 (1981)

10 Milton, G W, McPhedran, R C : A comparison of two methods for deriving bounds on the effective conductivity of composites In: Lecture Notes in Physics Vol 154 Berlin, Heidelberg, New York: Springer 1982, pp 183-193

11 Hashin, Z, Shtrikman, S : A variational approach to the theory of effective magnetic permeability of multiphase materials J Appl Phys 33, 3125-3131 (1962)

12 Hashin, $\mathrm{Z}$ : Theory of composite materials, in Mechanics of Composite Materials Wendt, F W, Lebowitz, H , Perrone, N (eds) New York: Pergamon Press 1970, pp 201-242

13 Beran, M : Statistical continuum theories, New York: Wiley 1968

14 Kohler, W , Papanicolaou, G : Bounds for the effective conductivity of random media In: Lecture Notes in Physics Vol 154 Berlin, Heidelberg, New York: Springer 1982, pp 111-130

15 Willis, J R : Bounds and selfconsistent estimates for the overall properties of anisotropic composites, J Mech Phys Solids, 25, (1977), pp 185-202

16 Willis, J R : Variational and related methods for the overall properties of composites, Advances in Applied Mechanics, Vol 21, New York: Academic Press, 1981

17 Dedericks, P H , Zeller, R : Variational treatment of elastic constants of disordered materials Z Phys 259, 103-116 (1973)

18 Papanicolaou, G, Varadhan, S : Boundary value problems with rapidly oscillating random coefficients (Colloquia Mathematica Societatis János Bolyai 27, Radom Fields, Esztergom (Hungary) 1979), Amsterdam: North Holland 1982, pp 835-873

19 Akhiezer, N I, Glazman, I M : The theory of linear operators in Hilbert space New York: F Ungar Publ Co 1966

20 Akhiezer, N I : The classical moment problem New York: Hafner 1965

21 Keller, J B : A theorem on the conductivity of a composite medium J Math Phys 5, 548-549 (1964)

22 Schulgasser, $\mathrm{K}:$ On a phase interchange relationship for composite materials $J$ Math Phys 17, 378 (1976)

23 Karlin, S , Studden, W J : Tchebycheff systems: with applications in analysis and statistics New York: John Wiley and Sons 1966

Communicated by J L Lebowitz

Received February 23, 1983 
